\title{
Comunicação Entre a Pesquisa Básica, Aplicada e Teórica na Análise do Comportamento no Brasil: Uma Análise Bibliométrica
}

\author{
Bruno Angelo Strapasson ${ }^{* a, b}$, Fernanda Gutierrez Magalhães ${ }^{b, c}$ \& Joice Kalfeld Custódio ${ }^{b}$ \\ ${ }^{\mathrm{a}}$ Universidade de São Paulo, São Paulo, Brasil, ${ }^{\mathrm{b}}$ Universidade Positivo, Curitiba, Brasil \& ${ }^{\mathrm{c}}$ Pontifícia Universidade \\ Católica de São Paulo, São Paulo, Brasil
}

\begin{abstract}
RESUMO
Muitos autores sugerem que deve haver alto grau de interação entre pesquisa aplicada, básica e teórica para um desenvolvimento adequado da Análise do Comportamento. Pretendeu-se aqui avaliar a comunicação entre essas áreas, mediante análise de referências de três publicações brasileiras. As revistas tiveram seus artigos e referências categorizados como aplicados, básicos e teóricos. Foram identificados 132 artigos teóricos, 90 aplicados e 43 básicos. Verificou-se a porcentagem de autorreferências e de referências cruzadas em cada categoria. Nos teóricos, as autorreferências são predominantes e nos aplicados, as referências cruzadas o são. Nos básicos, o número de autorreferências e referências cruzadas é similar. Observou-se um decréscimo das autorreferências e aumento das referências cruzadas nos últimos anos.
\end{abstract}

Palavras-chave: pesquisa aplicada; pesquisa básica; pesquisa teórica; análise do comportamento; análise bibliométrica.

\section{ABSTRACT \\ Communication Between Basic, Applied, and Theoretical Research in Behavior Analysis in Brazil: A Bibliometrical Analysis}

Many authors suggest that there should be high interaction between applied, basic and theoretical research for an adequate development of Behavior Analysis. The purpose of this text is to evaluate the communication between these areas, through the analysis of citations from three Brazilian publications. The journals have had their articles and references categorized as applied, basic and theoretical. One hundred thirty-two articles were sorted as theoretical, ninety as applied and fortythree as basic. The percentage of self-citation and cross-citation in each category was identified: The self-citations are predominant in theoretical articles, whereas cross-citations are more frequent in applied ones. In basic research texts, the number of self-citations is similar to cross-citations. It was observed a recent decrease in self-citations and an increase in cross-citations.

Keywords: applied research; basic research; theoretical research; behavior analysis; bibliometrical analysis.

Muitas tentativas de caracterização da Análise do Comportamento (AC) como uma ciência composta por diferentes áreas estão presentes na literatura (Carvalho Neto, 2002; Hayes, 1978; Lattal, 2005; Moore \& Cooper, 2003; Tourinho, 1999, 2003). De modo geral, entende-se que como um empreendimento científico a AC seria formada por ao menos três áreas essenciais: (1) a Análise Experimental do Comporta- mento (AEC), (2) a Análise Aplicada do Comportamento (AAC) e (3) o Behaviorismo Radical ${ }^{1}$ (BR); essas áreas seriam informadas pela pesquisa básica, pesquisa aplicada e pesquisa histórica/filosófica/conceitual (doravante, pesquisa teórica), respectivamente.

Também se encontram na literatura diversas indicações de que para um desenvolvimento adequado da $\mathrm{AC}$ as áreas citadas deveriam interagir fortemente e

Endereço para correspondência: Bruno Angelo Strapasson - brunoastr@gmail.com 
reciprocamente (Epling \& Pierce, 1983; Epling \& Pierce, 1986; Hake, 1982; Harzem, 2005; Lattal, 2005; Mace, 1994; Mace \& Wacker, 1994; Machado, Lourenço e Silva, 2000; Moxley, 1989; Pierce \& Epling, 1980; Sidman, 2011; Tourinho, 2003). Ainda que não seja consenso que analistas do comportamento envolvidos em pesquisa básica devam necessariamente se reportar às produções de autores envolvidos com pesquisas aplicadas ou teóricas ou que ocorra a recíproca (e.g., Baer, 1981; Poling et al., 1981), dificilmente algum analista do comportamento se oporia a tal interação. Além disso, a necessidade da interação entre essas áreas vem ganhando destaque nas discussões contemporâneas sobre pesquisas translacionais na AC (e.g., Critchfield, 2011; Mace \& Critchfield, 2010; Poling, 2010).

Entretanto, isso parece não ser, de fato, o que tem acontecido. Hayes, Rincover e Solnick (1980), por exemplo, indicam que há um decréscimo preocupante das publicações conceituais já nos primeiros números do Journal of Applied Behavior Analysis (JABA). De modo similar, Machado, Lourenço e Silva (2000) e Tourinho (2003) criticam uma aparente preponderância da pesquisa básica e aplicada em relação à pesquisa teórica e Deitz (1978), Michael (1980) e Pierce e Epling (1980) sugerem que há certo distanciamento da pesquisa básica em relação à pesquisa aplicada.

Um modo interessante de analisar empiricamente a comunicação ou distanciamento entre essas áreas é a aplicação de métodos de análise de referências na literatura pertinente. A análise de referências é um procedimento que tem se tornado cada vez mais comum nos diferentes âmbitos da ciência (Cronin, 2001) e que permite avaliar empiricamente a comunicação entre pesquisadores e influência intelectual (Critchfield, 2002; Garfield, 1970, 1972).

$\mathrm{Na} \mathrm{AC}$, estratégias bibliométricas de avaliação das referências foram usadas com vários propósitos. As tendências nas publicações de jornais da área (Coleman \& Mehlman, 1992; Krantz, 1971; Laties \& Mace, 1993; Serrano \& Ribes-Iñesta, 2007), a influência de textos da AC na própria comunidade de analistas do comportamento ou na comunidade externa (Dymond, O'Hora, Whelan \& O'Donovan, 2006; Eshleman, 1991; Kazdin, 1975) e a identificação de padrões de comunicação e de influência intelectual (Critchfield, 2002; Critchfield et al., 2000; Perone, 1985) são exemplos de temas abordados por meio da verificação quantitativa das referências de livros e periódicos renomados na $\mathrm{AC}$ e de outras áreas do conhecimento.

Um modo especialmente interessante de avaliação da comunicação entre duas áreas de pesquisa é a verificação da proporção de referências diretas a artigos de uma área de pesquisa em relação ao total de referências de um corpo definido de textos. Poling, Alling e Fuqua (1994) avaliaram desse modo a comunicação entre a pesquisa básica e aplicada na AC entre os anos de 1983 e 1992. Tomando o Journal of the Experimental Analysis of Behavior (JEAB) como o corpo de textos que representava a pesquisa básica e o Journal of the Applied Behavior Analysis (JABA) como o corpo de textos que representava a pesquisa aplicada, os autores avaliaram as proporções de autorreferência (porcentagem de referências nos artigos do JABA a artigos do próprio JABA e porcentagem de referências nos artigos do JEAB a artigos do JEAB) e referências cruzadas (porcentagem de referências a artigos do JEAB nos artigos do JABA e porcentagem de referências nos artigos do JEAB a artigos do JABA) desses jornais. Poling et al. (1994) constataram que, apesar de tanto o JEAB como o JABA apresentarem altos índices de autorreferência $(36,1 \%$ e $22,6 \%$, respectivamente), baixos índices de referências cruzadas foram encontrados em ambos os periódicos $(0,6 \%$ e $2,4 \%$, respectivamente). Esses resultados foram interpretados como indicadores de pouca comunicação entre a pesquisa básica e aplicada nos principais periódicos que vinculavam a produção em $\mathrm{AC}$ no período estudado.

Posteriormente, Elliot et al. (2005) atualizaram o levantamento de Poling et al. (1994), estendendo o período de análise para os anos de 1993 a 2003. Nessa atualização foram encontrados $32,2 \%$ e $34,9 \%$ de autorreferências, $0,6 \%$ e 7,8\% de referências cruzadas para JEAB e JABA, respectivamente. Na discussão do artigo, Elliot et al. (2005) identificaram uma estabilidade nas porcentagens de autorreferência e nas referências cruzadas do JEAB e aventaram a possibilidade de a alteração na porcentagem de referências cruzadas do JABA ser fruto das medidas editorias tomadas pelo jornal para aumentar a comunicação entre as áreas básica e aplicada. Utilizando o mesmo tipo de abordagem metodológica, Perone (1985) investigou os padrões assimétricos de referências cruzadas nas pesquisas experimentais com humanos e animais no JEAB sugerindo que a pesquisa com humanos exerce pouca influência nas pesquisas com animais. Strapasson e 
Carrara (2010) realizaram avaliações similares no JEAB e no JABA investigando a comunicação entre as pesquisas básica e aplicada sobre o "prestar atenção" e relataram menor grau de comunicação que aquele encontrado em Poling et al. (1994) e Elliot et al. (2005), a saber, $1,96 \%$ e $0,04 \%$ nas referências cruzadas do JEAB e JABA, respectivamente. Em seu conjunto, os estudos mencionados sugerem que a análise de referências vem se mostrando uma ferramenta empírica interessante na avaliação da comunicação entre áreas de pesquisa. Além disso, evidencia-se que apesar de a interação entre pesquisadores das áreas básica e aplicada ser desejável, ela vem ocorrendo em proporção insatisfatória.

O Brasil é um dos principais países no cenário mundial em AC, sendo que 13,6\% de seus psicólogos dizem atuar com uma "abordagem comportamentalista" (Gondin, Bastos \& Peixoto, 2010). Entretanto não há levantamentos sobre a interação entre a pesquisa básica, aplicada e teórica nesse país. O presente texto constitui uma tentativa de avaliar, em âmbito nacional, a comunicação entre essas três áreas consideradas como essenciais para o progresso da AC.

\section{MÉTODO}

Para avaliar a comunicação entre as pesquisas básicas, aplicadas e teóricas na literatura brasileira da AC foram acessados todos os números publicados da Revista Brasileira de Terapia Comportamental e Cognitiva (RBTCC, 1999-2011), da Revista Brasileira de Análise do Comportamento (REBAC, 20042008) e da revista Perspectivas em Análise do Comportamento (PAC, 2010-2011) até o final do ano de 2011. Os artigos presentes nessas revistas foram separados em quatro categorias (pesquisa aplicada, pesquisa básica, pesquisa teórica e outros) segundo os seguintes critérios:

Pesquisa aplicada - Artigos que disponham dados empíricos obtidos por observação direta ou indireta do comportamento (e.g. dados obtidos por meio de questionários, manipulação experimental em ambiente controlado, estudos de caso etc.) e tenham como objetivo genérico demonstrar a eficácia de procedimentos de intervenção ou produzir resultados úteis para resolução de problemas aplicados. Exemplos de pesquisas dessa categoria são avaliações da eficácia de tratamentos terapêuticos, estudos de caso ilustrando e aplicação de uma técnica específica e desenvolvimento de programas de leitura baseados em conceitos comportamentais, como os procedimentos de estabelecimento de equivalência de estímulos, ou mesmo a identificação de fontes de controle para operantes verbais em pessoas com déficits severos na fala.

Pesquisa básica - Artigos que disponham dados empíricos obtidos por observação direta ou indireta do comportamento (e.g. dados obtidos por meio de questionários, observação, manipulações experimentais em laboratório etc.) e tenham como objetivo o foco na descoberta de regularidade de eventos ou no teste de teorias e conceitos, sem interesse aplicado imediato. Exemplos de pesquisas básicas são avaliações de desempenhos de escolha em diferentes arranjos de estímulos, teste de diferentes arranjos experimentais para o estabelecimento de equivalência de estímulos ou avaliação das condições que aumentam ou diminuem a probabilidade de correspondência entre comportamento verbal e não verbal em experimentos em laboratório.

Pesquisa teórica - Artigos voltados para discussões conceituais e/ou filosóficas ou artigos voltados para discussões de dados da pesquisa básica e aplicada, mas que não descrevam dados obtidos por observação direta ou indireta do comportamento (i.e. textos que apenas compilem ou analisem dados obtidos por outras pesquisas). Exemplos dessa categoria são os artigos de revisão da literatura, traduções comportamentais de conceitos de outras teorias e outros tipos de discussão filosófica ou conceitual.

Outros - Artigos que não se enquadrem nas categorias anteriores. Exemplos de artigos dessa categoria são: textos que reproduzam decisões de entidades acadêmicas (e.g. consenso latino-americano de obesidade), obituários, artigos técnicos (e.g. estabelecimento de procedimentos, validação de instrumentos de pesquisa etc.), dicionários, biografias, obras literárias, testes psicológicos etc.

Para a classificação dos artigos nessas categorias foram acessados os títulos e resumos de todos os artigos. Nos casos em que os avaliadores não foram capazes de classificar a pesquisa apenas pelo resumo, consultou-se o texto completo para se obter mais elementos que permitissem a classificação. Em todos os casos em que, após esses procedimentos, ainda restaram dúvidas, pelo menos três classificadores leram o artigo e discutiram sua classificação até se chegar a um consenso.

As listas de referência de todos os artigos analisados, exceto aqueles classificados na categoria "ou- 
tros", foram consultadas e os textos lá referenciados foram também classificados segundo as categorias já apresentadas. Nessa etapa, as classificações foram feitas especialmente pelo título e para a verificação sobre se o artigo envolvia ou não coleta de dados originais, os resumos dos artigos referenciados foram procurados na internet. Caso não fossem encontrados os resumos ou estes não fossem suficientemente informativos a classificação da referência como básica, aplicada ou teórica era feita a partir de dados indiretos como (A) o trecho (e seu contexto) do texto avaliado em que a referência aparecia, (B) o veículo de publicação do texto (título do livro no caso de capítulos de livro e título do periódico, no caso de artigos) e (C) outras citações feitas ao texto referenciado, mas que estavam disponíveis apenas em outras fontes de informação (i.e. outros artigos científicos que não foram objetos de análise nesta pesquisa, mas que estavam disponíveis na internet). Como exemplo de informação indireta a partir do contexto da citação no texto (A), pode-se citar uma referência que aparecia em um contexto em que se estavam indicando diferentes resultados de pesquisa (e.g. "esse efeito também foi encontrado em humanos (Silva, 1998)"), nesse caso se excluía a possibilidade de pesquisa teórica. A análise do trecho sempre levava em conta outras referências citadas para ilustrar o mesmo aspecto que a referência em questão ilustrava e o conteúdo do parágrafo em que ela era citada. Já a informação deduzida do veículo de publicação do texto (B) pode ser exemplificada por referências de artigos que estavam publicadas no JEAB. Nesse caso, aumentava-se a probabilidade de considerá-lo uma pesquisa básica ou teórica. Similarmente, se o artigo estava publicado em um livro de terapia comportamental, aumentava-se a probabilidade de ser classificado como aplicado ou teórico, etc. A análise do título do veículo de publicação sempre levava em consideração as políticas editoriais dos periódicos avaliados, bem como eventuais resenhas disponíveis sobre os livros que continham os capítulos referenciados. No caso dos demais textos encontrados que citavam a mesma referência em análise (estratégia C), as mesmas verificações de contexto identificadas em (A) foram aplicadas. Em todos os casos que se apresentavam dificuldades na classificação pelo menos três avaliadores discutiram como classificar a referência até obter-se consenso.

Foram utilizadas como medidas da comunicação entre as áreas de pesquisa a porcentagem de referências ao mesmo tipo de texto (referências de textos teóricos a textos teóricos, de textos de pesquisa básica a pesquisas básicas e de textos de pesquisa aplicada à pesquisa aplicada) e a textos de tipos diferentes (referências de textos aplicados e teóricos em pesquisas básicas e assim por diante). Além disso, foi feita uma análise da porcentagem de autorreferência e referências cruzadas por tipo de pesquisa ao longo dos anos. Para a identificação dos textos mais citados as diferentes versões dos textos (e.g. diferentes edições ou traduções) foram consideradas como sendo o mesmo texto e apenas a data da publicação original foi considerada.

\section{RESULTADOS E DISCUSSÃO}

No total foram avaliados 265 artigos e a Tabela 1 indica a distribuição das categorias nas quais os artigos foram classificados. É possível perceber na tabela que o periódico com maior proporção de artigos da área aplicada é a RBTCC, a REBAC apresentou maior proporção de pesquisa básica e a PAC apresentou maior proporção de artigos teóricos. Tal padrão é coerente com as políticas editoriais e sugere certa especialização dessas revistas. Percebe-se também que aproximadamente metade dos artigos publicados em AC no Brasil não apresentam dados empíricos obtidos por observação direta ou indireta do comportamento.

Tabela 1

Distribuição dos Artigos por Categoria em Valores Brutos e Porcentagem

\begin{tabular}{cccccccc}
\hline & \multicolumn{2}{c}{ Aplicada } & \multicolumn{2}{c}{ Básica } & \multicolumn{2}{c}{ Teórica } & \multirow{2}{*}{ Total } \\
& $\mathrm{n}^{\text {o }}$ & $\%$ & $\mathrm{n}^{\text {o }}$ & $\%$ & $\mathrm{n}^{\text {o }}$ & $\%$ & \\
RBTCC & 82 & $42,7 \%$ & 18 & $9,4 \%$ & 92 & $47,9 \%$ & 192 \\
REBAC & 8 & $15,1 \%$ & 25 & $47,2 \%$ & 20 & $37,7 \%$ & 53 \\
PAC & 0 & $0,0 \%$ & 0 & $0,0 \%$ & 20 & $100,0 \%$ & 20 \\
\hline Total & 90 & $34,0 \%$ & 43 & $16,2 \%$ & 132 & $49,8 \%$ & 265 \\
\hline
\end{tabular}


A análise das publicações por tipo de artigo ao longo do tempo (ver Figura 1) mostra que o número de artigos aplicados varia entre 3 e 11 por ano, o de artigos básicos varia entre 0 e 11 e que entre os teóricos a variação vai de 4 a 19 . Ou seja, a variação é menor entre os artigos aplicados e dentre esses não se observam picos como nos outros dois tipos de pesquisa. Pode-se relacionar esse dado com o fato de a RBTCC ter sido publicada ininterruptamente por todo o período avaliado. Já no caso das pesquisas básicas, houve um pico de 2005 a 2008, período em que foram publi- cados os números da REBAC, revista direcionada para a pesquisa básica. Com a suspensão temporária da publicação da REBAC devido a dificuldades administrativas, a quantidade de pesquisas básicas caiu consideravelmente. No que se refere às pesquisas teóricas, há um pico no ano da primeira publicação da REBAC, em que foram publicados 12 artigos teóricos na RBTCC e sete na REBAC. Entre 2006 e 2009 o número de artigos teóricos permanece entre 7 e 14 e volta a crescer com o lançamento da revista Perspectivas em AC, revista voltada para publicações teóricas.

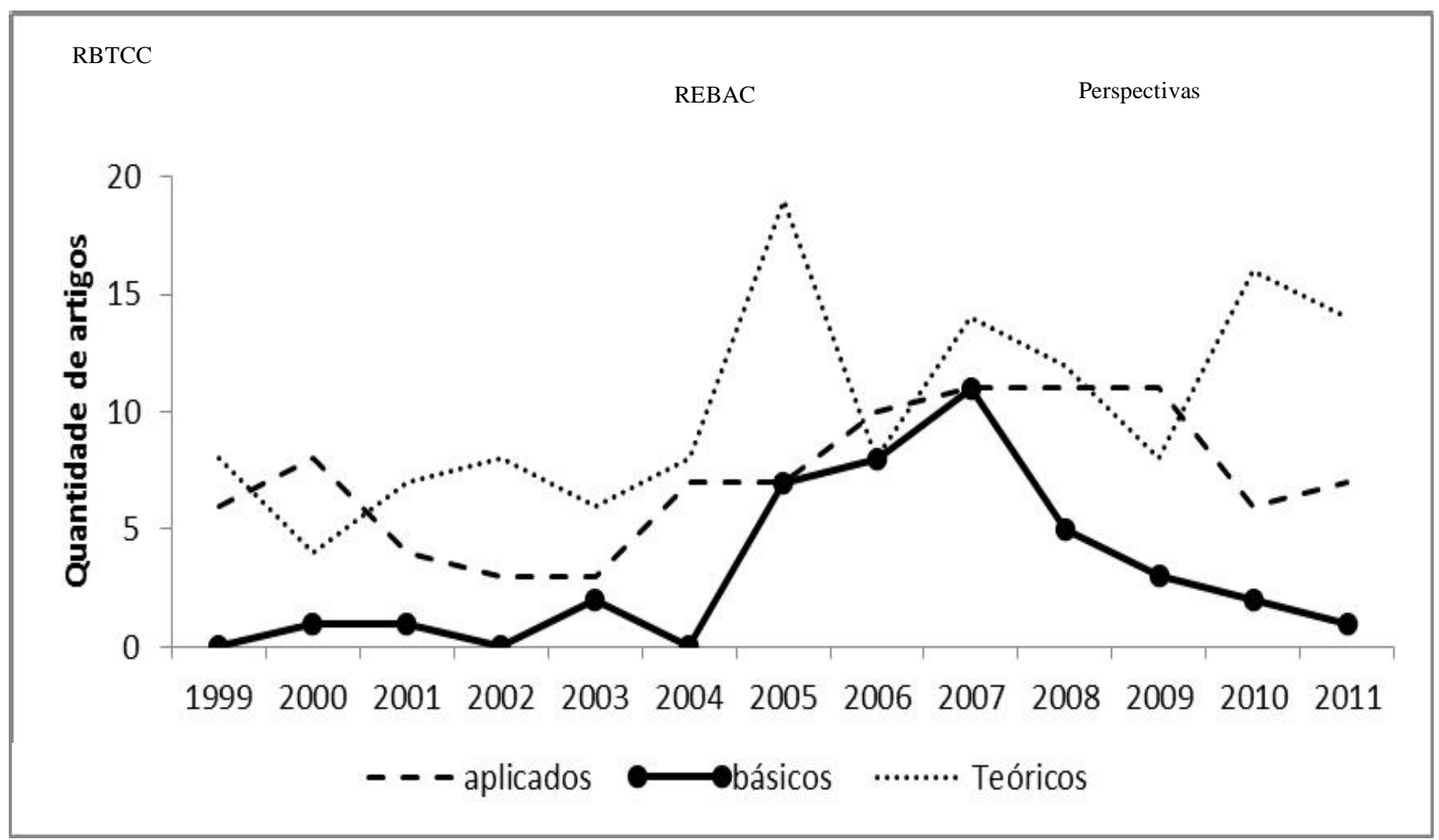

Figura 1. Distribuição temporal dos artigos de pesquisa básica, aplicada e teórica comparados aos períodos de publicação dos periódicos avaliados.

Dos 265 artigos avaliados foram verificadas 7332 referências sendo, portanto, 27,66 referências por artigo, em média. Essas referências se reportam a 5698 diferentes textos. Aproximadamente dois terços dessas referências $(63,84 \%)$ se referiam a textos em outros idiomas que não o português ou a textos traduzidos. Ainda que não existam parâmetros para avaliar quão expressiva é essa proporção, percebe-se que a literatura nacional já mantém certa independência da literatura internacional no sentido de se estabelecer como fonte de consulta.

\section{Autorreferência e referências cruzadas}

Os índices de autorreferência apresentaram-se como $29,4 \%, 48,6 \%$ e $72,6 \%$ para a pesquisa aplicada, básica e teórica, respectivamente. No caso das referências cruzadas percebem-se os seguintes padrões: dos artigos classificados como pesquisa aplicada apenas 7,3\% das referências eram de pesquisas básicas e $57,5 \%$ de artigos teóricos. Os autores de artigos classificados como pesquisa básica referenciaram outros textos da área aplicada em $16,9 \%$ de todas as referências e a artigos da área teórica em 32,7\%. Das referências dos artigos teóricos, $12,5 \%$ eram de pesquisas aplicadas e $11,6 \%$ de pesquisas básicas. A Tabela 2 
resume os dados de autorreferência e referências cruzadas da pesquisa aplicada, básica e teórica nas dife- rentes revistas analisadas bem como sua análise em conjunto.

Tabela 2

Autorreferência e Referências Cruzadas por Categoria em Números Totais e Porcentagem

\begin{tabular}{|c|c|c|c|c|c|c|c|c|c|c|}
\hline \multirow{2}{*}{ Revista } & \multirow{2}{*}{$\begin{array}{c}\text { Tipo } \\
\text { pesquisa }\end{array}$} & \multicolumn{2}{|c|}{$\begin{array}{l}\text { Referências } \\
\text { Aplicadas }\end{array}$} & \multicolumn{2}{|c|}{$\begin{array}{l}\text { Referências } \\
\text { Básicas }\end{array}$} & \multicolumn{2}{|c|}{$\begin{array}{l}\text { Referências } \\
\text { Teóricas }\end{array}$} & \multicolumn{2}{|c|}{$\begin{array}{l}\text { Referências } \\
\text { outros }\end{array}$} & \multirow[t]{2}{*}{ Total } \\
\hline & & $\mathrm{n}^{\mathrm{o}}$ & $\%$ & $\mathrm{n}^{\mathrm{o}}$ & $\%$ & $\mathrm{n}^{\mathrm{o}}$ & $\%$ & $\mathrm{n}^{\mathrm{o}}$ & $\%$ & \\
\hline \multirow[t]{4}{*}{ RBTCC } & Aplicada & 511 & 27,6 & 125 & 6,8 & 1105 & 59,7 & 110 & 5,9 & 1851 \\
\hline & Básica & 140 & 24,6 & 222 & 39,1 & 191 & 33,6 & 15 & 2,6 & 568 \\
\hline & Teórica & 386 & 14,6 & 228 & 8,6 & 1950 & 73,6 & 84 & 3,2 & 2648 \\
\hline & total & 1037 & 20,5 & 575 & 11,3 & 3246 & 64,1 & 209 & 4,1 & 5067 \\
\hline \multirow[t]{4}{*}{ REBAC } & Aplicada & 100 & 44,1 & 26 & 11,5 & 94 & 41,4 & 7 & 3,1 & 227 \\
\hline & Básica & 75 & 10,7 & 396 & 56,3 & 224 & 31,9 & 8 & 1,1 & 703 \\
\hline & Teórica & 33 & 4,6 & 122 & 17 & 543 & 75,7 & 19 & 2,6 & 717 \\
\hline & total & 208 & 12,6 & 544 & 33 & 861 & 52,3 & 34 & 2,1 & 1647 \\
\hline \multirow[t]{4}{*}{ PAC } & Aplicada & 0 & 0 & 0 & 0 & 0 & 0 & 0 & 0 & 0 \\
\hline & Básica & 0 & 0 & 0 & 0 & 0 & 0 & 0 & 0 & 0 \\
\hline & Teórica & 80 & 12,9 & 112 & 18,1 & 397 & 64,2 & 4,7 & 4,7 & 618 \\
\hline & total & 80 & 12,9 & 112 & 18,1 & 397 & 64,2 & 4,7 & 4,7 & 618 \\
\hline \multirow{4}{*}{$\begin{array}{l}\text { Total } \\
\text { geral }\end{array}$} & Aplicada & 611 & 29,4 & 151 & 7,3 & 1199 & 57,7 & 117 & 5,6 & 2078 \\
\hline & Básica & 215 & 16,9 & 618 & 48,6 & 415 & 32,7 & 23 & 1,8 & 1271 \\
\hline & Teórica & 499 & 12,5 & 462 & 11,6 & 2890 & 72,6 & 132 & 3,3 & 3983 \\
\hline & total & 1325 & 18,1 & 1231 & 16,8 & 4504 & 61,4 & 272 & 3,7 & 7332 \\
\hline
\end{tabular}

A análise da porcentagem de autorreferências nos três tipos de pesquisa ao longo do tempo mostra, entre os anos de 1999 e 2005, variação de 19,6\% para as pesquisas aplicadas, de 17,9\% para as pesquisas básicas e $16,2 \%$ para os artigos teóricos. Adicionalmente pode-se identificar um decréscimo constante, nos últimos cinco anos, exceto para o ano de 2010 no caso das pesquisas básicas, como mostra a Figura 2. Todavia, a porcentagem de autorreferências é maior nas pesquisas básicas e teóricas. O número de autorreferências em pesquisas aplicadas decresce nos primeiros cinco anos, volta a crescer nos três anos seguintes e permanece com tendência descendente desde 2005, ocupando a faixa entre $30 \%$ e $40 \%$ de todas as referências citadas. Já nas pesquisas básicas, a ocorrência de autorreferências é relativamente baixa nos primeiros anos. Em 1999, 2002 e 2004, não há publicação de artigos de pesquisa básica. Em 2003, é alta a ocorrência de autorreferências, mantendo-se alta nos últimos cinco anos, variando entre $40 \%$ e $65 \%$ do total de referências. Considerando as autorreferências em pesquisas teóricas, percebe-se que, desde o início do período investigado, a porcentagem é superior a $50 \%$ e tem se mantido entre $70 \%$ e $80 \%$ nos últimos cinco anos. 


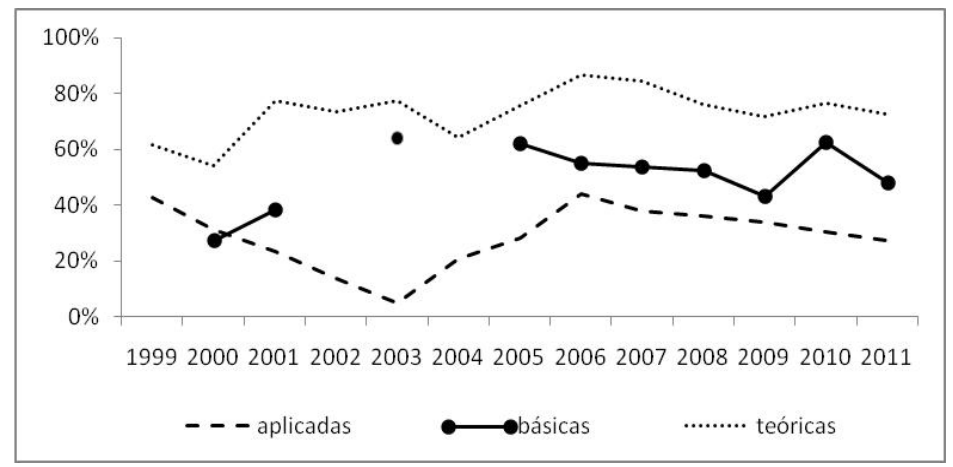

Figura2. Distribuição temporal das porcentagens de autorreferência dos artigos de pesquisa básica, aplicada e teórica. Nos anos de 1999, 2002 e 2004 não foram publicados artigos de pesquisa básica nos periódicos analisados.

A análise da porcentagem de referências cruzadas mostra aumento nos cinco últimos anos nos três tipos de pesquisa, como pode ser visto na Figura 3. Entretanto, as pesquisas aplicadas apresentam uma porcentagem maior de referências cruzadas do que os outros dois tipos de pesquisa. Enquanto as referências cruza- das em pesquisas aplicadas ocupam entre $60 \%$ e $70 \%$ de todas as referências citadas nos últimos cinco anos, no caso da pesquisa básica, as referências cruzadas ficam entre $40 \%$ e $60 \%$ e no caso das pesquisas teóricas, entre $20 \%$ e $30 \%$.

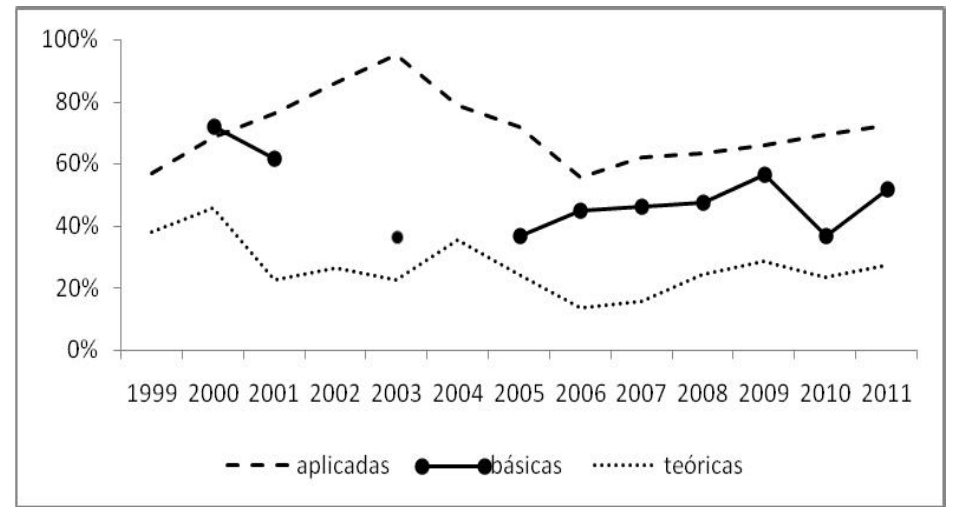

Figura 3. Distribuição temporal das porcentagens de referências cruzadas dos artigos de pesquisa básica, aplicada e teórica. Nos anos de 1999, 2002 e 2004 não foram publicados artigos de pesquisa básica nos periódicos analisados.

Os dados sugerem que diferentemente das tendências levantadas por outros autores (Hayes et al., 1980; Machado et al., 2000; Tourinho, 2003), nos periódicos estudados há um número mais elevado de pesquisas teóricas que aplicadas. Levando em consideração a diferença entre pesquisas empíricas (básicas e aplicadas) e teóricas (históricas/filosóficas/conceituais), as quantidades são similares (133 e 132, respectivamente).

Sobre a necessidade de interação entre as áreas de pesquisa, a análise de autorreferência e referências cruzadas mostrou um cenário de pouca interação no caso das pesquisas teóricas nos últimos anos, quando as autorreferências ultrapassaram os $70 \%$. Com relação às pesquisas aplicadas e básicas, a frequência de referências cruzadas é alta, entretanto a maior parte se refere a textos teóricos $(57,7 \%$ nas pesquisas aplicadas e 32,7 nas básicas). Já a análise ao longo do tempo mostra uma tendência de diminuição das autorreferência e incremento das referências cruzadas nas três áreas de pesquisa, o que pode indicar um prognóstico de maior interação, condizente com o comumente prescrito para um desenvolvimento adequado da AC.

\section{Textos e periódicos mais citados:}

Dos onze textos mais citados nos periódicos consultados seis são textos de Skinner (Skinner, 1953/1965, 1957, 1969, 1974, 1981, 1989) e apenas o décimo primeiro lugar não tem tradução para o português. A Tabela 3 mostra os onze textos mais citados. 
Tabela 3

Textos Mais Citados

\section{Texto}

Skinner, B. F. (1953) Ciência e comportamento humano

Skinner, B. F. (1957) O comportamento verbal

Skinner, B. F. (1974) Sobre o Behaviorismo

Catania, A. C. (1998) Aprendizagem, comportamento, linguagem e cognição

Skinner, B. F. (1969) Contingências de reforço: Uma análise teórica

Skinner B. F. (1981) Seleção por consequências

Sidman, M. (1989) Coerção e suas implicações

Kohlemberg, R. J \&Tsai, M. (1991) Psicoterapia analítico-funcional

Skinner, B. F. (1989) Questões recentes na análise do comportamento

Skinner, B. F. (1945) The operational analysis of psychological terms

Sidman, M. \& Tailby, W. (1982) Conditional discrimination vs. matching to sample: An expansion of the testing paradigm
Quantidade de referências

90

87

65

51

31

31

27

25

22
Dos textos de autoria de brasileiros, os mais citados foram "Relação terapêutica" (Meyer \& Vermes, 2001) e "O relato verbal segundo a perspectiva da análise do comportamento: contribuições conceituais e experimentais" (DeRose, 1997), ambos textos teóricos.

Analisando-se os periódicos mais citados, pode-se perceber que dentre os dez mais frequentes apenas três são nacionais. Destes, ganha destaque a coleção Sobre Comportamento e Cognição, que apesar de não se submeter aos padrões tradicionais de avaliação da qualidade e revisão por pares, parece ter se tornado uma das principais fontes de informação na $\mathrm{AC}$ no Brasil. Em segundo lugar, a revista Psicologia: Teoria e Pesquisa figura como uma importante fonte de informação. Apesar de essa revista apresentar uma política editorial abrangente sem privilegiar a publicação de textos de analistas do comportamento, ela acabou se constituindo um veículo de publicação importante para os behavioristas especialmente antes da criação da RBTCC.

No caso dos periódicos internacionais, o Journal of the Experimental Analysis of Behavior e o Journal of Applied Behavior Analysis são as principais fontes de informação compondo juntos quase $10 \%$ de todas as referências analisadas. 
Tabela 4

Principais Periódicos Citados

\begin{tabular}{lc}
\multicolumn{1}{c}{ Periódico } & $\begin{array}{c}\text { Quantidade de } \\
\text { referências }\end{array}$ \\
Journal of the Experimental Analysis of Behavior & 500 \\
Coleção Sobre Comportamento e Cognição & 285 \\
Journal of Applied Behavior Analysis & 138 \\
Psicologia: Teoria e Pesquisa & 92 \\
The Behavior Analyst & 92 \\
The Analysis of Verbal Behavior & 88 \\
Revista Brasileira de Terapia Comportamental e Cognitiva & 78 \\
Psychological Record & 66 \\
American Psychologist & 52 \\
Behavior Therapy & 49 \\
\hline
\end{tabular}

\section{DISCUSSÃO GERAL}

Não é possível uma comparação direta desses resultados com outros estudos similares a este. Enquanto os estudos que analisaram a literatura estadunidense (Poling et al., 1994; Elliot et al., 2005) utilizaram jornais específicos como o corpo de textos que compõe o que foi considerado como pesquisa básica e aplicada, neste estudo cada artigo foi classificado como sendo um relato de pesquisa básica, aplicada ou teórica. Essa diferença se justifica porque, no Brasil, os jornais pesquisados apesar de terem políticas editorias que direcionam os tipos de pesquisa preferencialmente publicados, ainda não se estabeleceram como veículos prioritários para as diferentes áreas tal como atesta a Tabela 1. Além disso, as revistas pesquisadas vêm publicando continuamente artigos na área há pouco tempo de modo que dentre todos os artigos consultados as próprias revistas foram referenciadas poucas vezes - $78(1,21 \%), 13(0,20 \%)$ e zero $(0 \%)$ para a RBTCC, REBAC e PAC, respectivamente.

É preciso ponderar também a abrangência dos dados aqui apresentados. Esta avaliação bibliométrica investigou apenas os periódicos com declarada ênfase na publicação de textos embasados na AC no Brasil, entretanto, os autores nacionais têm frequentemente publicado em periódicos nacionais de psicologia com políticas editorias mais abrangentes e em periódicos de AC internacionais. Considerar essas publicações perpassa a difícil tarefa de criar critérios claros para se diferenciar artigos embasados na AC dentre os demais artigos publicados num periódico de psicologia geral, bem como de encontrar os textos de brasileiros em periódicos internacionais, mas poderá aprimorar a análise desenvolvida aqui.

Apesar dessas limitações, esta pesquisa levantou alguns dados que podem ser contrapostos aos observados na literatura internacional. Diferentemente da proposição de alguns autores (Hayes et al., 1980; Machado et al., 2000; Tourinho, 2003) de uma preponderância da pesquisa básica e aplicada em relação à pesquisa teórica, os dados levantados mostraram um número bem maior de pesquisas teóricas em relação às básicas e aplicadas. Além disso, a frequência de referências teóricas é alta nos três casos.

Autores como Epling e Pierce (1986), Hake (1982), Harzem (2005), Lattal (2005), Mace (1994), Moxley (1989), Machado et al. (2000), Sidman (2011) e Tourinho (2003) defendem a necessidade de um equilíbrio na produção de conhecimento entre a $\mathrm{AAC}$, a $\mathrm{AEC}$ e o BR. Machado et al. (2000) argumentam que sem dados factuais, as asserções conceituais e teóricas são vazias. Por outro lado, sem anaálises teóricas e conceituais, os fatos são cegos, desorganizados e sem sentido.

É possível verificar que a produção brasileira representada nas revistas avaliadas neste estudo mantém uma assimetria importante, na qual a publicação de pesquisas básicas e aplicadas é pequena e a publicação de artigos teóricos é grande. Há autores, porém, que não veem a preponderância de estudos teóricos sobre os demais como prejudicial. Serrano e Ribes-Iñesta (2007), ao avaliar a produção publicada na revista Acta Comportamentalia, consideram essa preponderância tão somente uma tradição diferente daquela observada nas revistas anglo-saxônicas, mas esse pa- 
drão não constituiria, necessariamente, uma prática negativa. Para avaliar em que medida essa assimetria nos tipos de pesquisa representa um déficit na produção de conhecimento ou apenas uma tradição diferente, uma análise do conteúdo das pesquisas seria necessária. Além disso, é preciso ponderar que, dados os critérios utilizados nesta pesquisa, não foram diferenciados artigos didáticos de artigos que produzem reflexões originais no campo teórico. Nessas condições, não é possível avaliar se a preponderância de artigos desse tipo representa uma acentuada preocupação com a investigação dos compromissos teóricos da $\mathrm{AC}$ ou se representa um esforço maior na produção de textos acessíveis a estudantes e interessados.

Outras perguntas surgem a partir dos dados obtidos nesta pesquisa. Por exemplo, mediante a queda no volume de pesquisas básicas identificada com a suspensão da publicação da REBAC, cabe perguntar se a publicação desse gênero de pesquisa de fato diminuiu no Brasil ou se migrou para outros periódicos. Lindsley (1987) e Skinner (1979) sugerem que os primeiros periódicos em AC dos Estados Unidos (JEAB e JABA) surgiram em decorrência da dificuldade para publicar pesquisas que recorriam ao delineamento de sujeito único em periódicos tradicionais de psicologia experimental. A origem de revistas especializadas em AC no Brasil aparentemente não foi a mesma, uma vez que é possível identificar artigos de AC em revistas generalistas de psicologia (e.g., Psicologia: Teoria e Pesquisa) mesmo antes do surgimento dos periódicos aqui analisados. Qual é o papel histórico dos periódicos especializados em $\mathrm{AC}$ no Brasil e em que medida eles regularam a publicação nacional são perguntas que fogem ao escopo das estratégias metodológicas utilizadas neste estudo, mas que permanecem a espera de novas investigações que possam respondê-las.

Por fim, mesmo considerando-se as limitações inerentes ao método utilizado nesta pesquisa é possível identificar algumas tendências, que suscitam perguntas e que merecem investigação sobre a produção de conhecimento em AC no Brasil. Investigar, por exemplo, se a assimetria entre os tipos de pesquisa é danosa ou se esta é apenas uma característica que distingue a produção brasileira de outras produções internacionais, poderia aprimorar a avaliação de prescrições para uma maior interação entre as áreas básica, aplicada e teórica. Avaliar se a existência de revistas vocacionadas para certo tipo de pesquisa aumenta a produção na área ou se apenas concentra a sua divulgação e quais os impactos desses efeitos na comunicação da AC com outras disciplinas científicas pode ser uma ferramenta interessante para se compreender a dinâmica de comunicação interdisciplinas bem como para se planejar políticas de fomento, visibilidade e comunicação científicas. Por fim, avaliar se os padrões aqui encontrados se repetem nos veículos de publicação não especializados (e.g. Psicologia: Teoria e Pesquisa, Interação em Psicologia, etc.) ou mesmo nos especializados, mas sem avaliação por pares (e.g., coleção Sobre Comportamento e Cognição, Comportamento em Foco, etc.) ajudará a avaliar a representatividade e significado dos resultados aqui encontrados. Pesquisas que objetivem responder essas questões permitirão uma compreensão mais acurada dos rumos da pesquisa em AC no Brasil.

\section{REFERÊNCIAS}

Baer, D. M. (1981). The flight of behavior analysis. The Behavior Analyst, 4, 85-91.

Carvalho Neto, M. B. (2002). Análise do comportamento: Behaviorismo radical, análise experimental do comportamento e análise aplicada do comportamento. Interação em Psicologia, 6, 13-18.

Catania, A. C. (1998). Learning ( $4^{\mathrm{a}}$ ed.). Upper Saddle River, NJ: Prentice Hall.

Coleman, S. R., \& Mehlman, S. E. (1992). An empirical update (1969-1989) of D. L. Krantz's thesis that the experimental analysis of behavior is isolated. The Behavior Analyst, 15, 4349.

Critchfield, T. S., Buskist, W., Saville, B., Crockett, J., Sherburne, T. \& Keel, K. (2000). Sources most frequently cited in the experimental analysis of human behavior. The Behavior Analyst, 23, 255-266.

Critchfield, T. S. (2002). Evaluating the function of applied behavior analysis: A bibliometric analysis. Journal of Applied Behavior Analysis, 35, 243-246.

Critchfield, T. S. (2011). Translational contributions of the experimental analysis of behavior. The Behavior Analyst, 34, 3-17.

Cronin, B. (2001). Bibliometrics and beyond: Some thoughts on web-based citation analysis. Journal of Information Science, 27, 1-7.

Deitz, S. M. (1978). Current status of applied behavior analysis: Science versus technology. American Psychologist, 33, 805814.

Dymond, S., O'Hora, D., Whelan, R., \& O'Donovan, A. (2006). Citation analysis of Skinner's Verbal Behavior. The Behavior Analyst, 29, 75-88.

Elliot, A. J., Morgan, K., Fuqua, W., Ehrardt, K., \& Poling, A. (2005). Self- and cross-citations in the Journal of Applied Behavior Analysis and the Journal of Experimental Analysis of Behavior: 1993-2003. Journal of Applied Behavior Analysis, 38, 559-563.

Interação Psicol., Curitiba, v. 17, n. 1, p. 117-128, jan./abr. 2013 
Epling, W. F., \& Pierce, W. D. (1983). Applied behavior analysis: New directions from the laboratory. The Behavior Analyst, 6, 27-37.

Epling, W. F., \& Pierce, W. D. (1986). Basic importance of applied behavior analysis. The Behavior Analyst, 9, 89-99.

Eshleman, J. W. (1991). Quantified trends in the history of verbal research. The Analysis of Verbal Behavior, 9, 61-80.

Garfield, E. (1970). Citation indexing for studying science. $\mathrm{Na}$ ture, 227, 669-671.

Garfield, E. (1972). Citation analysis as a tool in journal evaluation. Science, 178, 471-479.

Gondin, S. M. G., Bastos, A. V. B., \& Peixoto, L. S. A. (2010). Áreas de atuação, atividades e abordagens teóricas do psicólogo brasileiro. In A. V. B. Bastos \& S. M. G. Gondim (Eds.), $O$ trabalho do psicólogo no Brasil (pp. 174-199) Porto Alegre: ArtMed.

Hake, D. F. (1982). The basic applied continuum and the possible evolution of human operant social and verbal research. The Behavior Analyst, 5, 21-28.

Harzem, P. (2005). On the incongruence of theory and practice in behavioral research: What can and should be done about it. Mexican Journal of Behavior Analysis, 31, 85-95.

Hayes, S. C. (1978). Theory and technology in behavior analysis. The Behavior Analyst, 1, 25-33.

Hayes, S. C., Rincover, A., \& e Solnick, J. V. (1980). The technical drift of applied behavior analysis. Journal of Applied Behavior Analysis, 13, 275-285.

Kazdin, A. E. (1975). The impact of applied behavior analysis on diverse areas of research. Journal of Applied Behavior Analysis, 8, 213-229.

Kohlenberg, R. J., \& Tsai, M. (1991). Functional Analytic Psychotherapy: A guide for creating intense and curative therapeutic relationships. New York, NY: Plenum.

Krantz, D. L. (1971). The separate worlds of operant and non-operant psychology. Journal of Applied Behavior Analysis, 4, 61-70.

Laties, V. G., \& Mace, F. C. (1993). Taking stock: The first 25 years of the Journal of Applied Behavior Analysis. Journal of Applied Behavior Analysis, 26, 513-525.

Lattal, K. A. (2005). Ciência, tecnologia e análise do comportamento. In J. Abreu-Rodrigues \& M. R. Ribeiro (Eds.), Análise do comportamento: Pesquisa, teoria e aplicação (pp. 15-26). Porto Alegre: ArtMed.

Lindsley, O. L. (1987). Collecting the first dollars for JEAB. Journal of the Experimental Analysis of Behavior, 48, 469-471.

Mace, F.C. (1994). Basic research needed for stimulating the development of behavioral technologies. Journal of the Experimental Analysis of Behavior, 61, 529-550.

Mace, F. C., \& Critchfield, T. S. (2010). Translational research in behavior analysis: Historical traditions and imperative for the future. Journal of the Experimental Analysis of Behavior, 93, 293-312.

Mace, F. C., \& Wacker, D. P. (1994). Toward a greater integration of basic and applied behavioral research: An introduction. Journal of Applied Behavior Analysis, 27, 669-674.

Machado, A., Lourenço, O., \& Silva, F. J. (2000). Facts, concepts, and theories: The shape of psychology's epistemic triangle. Behavior and Philosophy, 28, 1-40.
Michael, J. (1980). Flight from behavior analysis. The Behavior Analyst, 3, 1-24.

Moore, J., \& Cooper, J. O. (2003). Some proposed relations among the experimental analysis of behavior, applied behavior analysis, and service delivery. The Behavior Analyst, 26, 6984.

Moxley, R. A. (1989). Some historical relationship between science and technology with implications for behavior analysis. The Behavior Analyst, 12, 45-57.

Perone, M. (1985). On the impact of human operant research: Asymmetrical patterns of cross-citation between human and nonhuman research. The Behavior Analyst, 8, 185-189.

Pierce, W. D., \& Epling, W. F. (1980). What happened to analysis in applied behavior analysis. The Behavior Analyst, 3, 1-9.

Poling, A. (2010). Looking to the future: Will behavior analysis survive and prosper? The Behavior Analyst, 33, 7-17.

Poling, A., Alling, K., \& Fuqua, W. (1994). Self- and cross-citations in the Journal of Applied Behavior Analysis and the Journal of Experimental Analysis of Behavior: 1983-1992. Journal of Applied Behavior Analysis, 27, 729-731.

Poling, A., Picker, M., Grossett, D., Hall-Johnson, E., \& Holbrook, M. (1981). The schism between experimental and applied behavior analysis: Is it real and who cares? The Behavior Analyst, 4, 93-102.

Serrano, M., \& Ribes-Iñesta, E. (2007). Acta Comportamentalia 1992-2006: Un análisis bibliométrico. Acta Comportamentalia, 15, 103-109.

Skinner, B. F. (1957). Verbal behavior. New York, NY: Appleton-Century-Crofts.

Skinner, B. F. (1965). Science and Human Behavior. New York, NY: Macmillan. (Originalmente publicado em 1953)

Skinner, B. F. (1974). About behaviorism. New York, NY: Alfred A. Knopf.

Skinner, B. F. (1979). The shaping of a behaviorist: Part two of an autobiography. New York, NY: Alfred A. Knopf.

Skinner, B. F. (1981). Selection by consequences. Science, 213, 501-504.

Skinner, B. F. (1989). Recent issues in the analysis of behavior. Columbus, OH: Merrill.

Sidman, M. (1989). Coercion and its fallout. Boston, MA: Authors Cooperative.

Sidman, M. (2011). Can an understanding of basic research facilitate the effectiveness of practitioners? Reflections and personal perspectives. Journal of Applied Behavior Analysis, 44, 973991.

Sidman, M., \& Tailby, W. (1982). Conditional discrimination vs. matching to sample: An expansion of the testing paradigm. Journal of the Experimental Analysis of Behavior, 37, 5-22.

Strapasson, B. A., \& Carrara, K. (2010). Comunicação entre JEAB e JABA na pesquisa sobre prestar atenção. In M. M. C. Hübner, M. R. Garcia, P. R. Abreu, E. N. P. Cillo e P. B. Faleiros (Eds.), Sobre comportamento e cognição: Vol. 25 (pp. 337343). Santo André, SP: ESETec.

Tourinho, E. Z. (1999). Estudos conceituais na análise de comportamento. Temas em Psicologia da SBP, 7, 213-222.

Tourinho, E. Z. (2003). A produção de conhecimento em psicologia: A análise do comportamento. Psicologia: Ciência e Profissão, 23, 30-41. 
Recebido: 09/04/2012

Última revisão: 07/01/2013

Aceito: $28 / 01 / 2013$

\section{Nota:}

1 Alguns autores incluem como um quarto elemento a tecnologia ou a prestação de serviços (e.g., Hayes, 1978). Entretanto, uma vez que na presente pesquisa optou-se por considerar estudos de caso como pesquisas aplicadas e a simples divulgação de organizações ou serviços não foi computada, entende-se que para os propósitos específicos deste texto a inclusão de uma quarta categoria seria desnecessária. Para o leitor interessado nas diferentes relações da prestação de serviços com a Análise do Comportamento enquanto uma disciplina científica indica-se a leitura de Lattal (2005). 